\title{
From the trajectory of heritability to the heritability of trajectories
}

\author{
Rogier A. Kievit ${ }^{1}$ \\ Jessica A. Logan ${ }^{2}$ \\ Sara A. Hart ${ }^{3,4}$
}

1. Cognitive Neuroscience Department, Donders Institute for Brain, Cognition and Behavior, Radboud University Medical Center, Nijmegen, The Netherlands

Rogier.kievit@radboudumc.nl

2. Department of Educational Studies, the Ohio State University, 29 W. Woodruff Ave, Columbus, OH, 43210, USA

3.
Department of Psychology, Florida State University, 1107 W. Call Street, Tallahassee, FL, 32308, USA
4. Florida Center for Reading Research, Florida State University, Tallahassee, FL, USA

\begin{abstract}
Although compelling and insightful, the proposal by Uchiyama et al. largely neglects within-person change over time, arguably the central topic of interest within their framework. Longitudinal behavioural genetics modeling suggests that the heritability of trajectories is low, in contrast to high and increasing cross-sectional heritability across development. Better understanding of the mechanisms of trajectories remains a crucial outstanding challenge.
\end{abstract}

\section{Main text}

In their target article, Uchiyama et al., argue for a nuanced, integrative perspective on understanding phenotypic variation as a function of cultural and genetic dynamics. Although compelling and insightful, arguably the most important source of dynamics is neglected: Withinperson change over time (Molenaar, 2004). Within-person changes such as slopes across waves capture how traits develop and grow over time. They have unique variance components and contributing factors distinct from baseline individual differences. In section 4.2, Uchiyama et al. describe the role of cultural heterogeneity on heritability across development, describing the behavioural genetics literature that exists on the "development" of traits - We scare quote development because the overwhelming majority of behavioural genetics work cited by Uchiyama et al. uses cross-sectional methods. However, many of their proposed mechanisms, such as geneenvironment interactions or the impact of (changing) cultural innovation operate within individuals over time. Ignoring within-person change is a crucial omission, as only under extreme and implausible circumstances (i.e. ergodicity, Molenaar, 2004) will cross-sectional inferences generalize to (longitudinal) processes within individuals. Although comparatively rare, longitudinal behaviour genetic studies of phenotypic change do exist, commonly studied by combining latent growth curve models with quantitative genetics decomposition, and suggest two key messages germane to the target article.

First, the heritability of within-person change is generally lower than that of baseline or static scores (e.g. Hatoum et al., 2018; Hart et al., 2013; Lyons et al. 2017; Reynolds et al., 2002). In other words, the process of learning, growing, and changing across the lifespan has a stronger environmental component than any one single measurement occasion. At the same time, age specific (i.e. crosssectional) heritability estimates of the same skills (e.g. reading) may increase across the lifespan - A distinct instance of Simpson's Paradox that further complicates our mechanistic understanding of phenotypic processes.

Second, to the extent that within-person changes (slopes) are heritable, empirical evidence suggests that the genetic influences on slopes are partially or wholly distinct from the genetic 
influences on baseline scores, as well as partially or wholly distinct between constructs (e.g. Finkel et al., 2013, Logan et al., 2013). This suggests there is meaningful variance on the within-person slope that is distinct from that of baseline scores: The genetic mechanisms affecting the baseline phenotype are not the same as those governing within person changes over time. In addition, the decreased heritability on the slope is not always associated with increased nonshared environmental influences. Together these findings demonstrate that the lower heritability estimates of slopes compared to intercepts cannot be explained away by the methodological challenges of measuring and estimating change over time, and as such reflect meaningful patterns that need to be reconciled in frameworks that hope to integrate both sources of phenotypic variation.

We believe these two key messages have at least three implications for the target article. First, we should be transparent about the source and locus of 'phenotypic variation'. Phenotypes vary between people, but also within people over time (called slopes or trajectories). When relying on cross-sectional evidence, we are drawing conclusions about how heritability changes across development; In other words, the target article studies the trajectory of heritability. This is distinct from the heritability of how a skill changes over time; The heritability of trajectories. When positing explanatory mechanisms, we must scrutinize whether the empirical evidence we rely on is suitable to provide evidence for the mechanism at hand. Within-person changes are governed by genetic influences and cultural exposure which differ across the lifespan within the same person. Differences in trajectories (and baseline-trajectory correlation) are arguably a primary source of increases, and decreases, in phenotypic variance and thus can affect heritability estimates across the lifespan. For such reasons, Finkel et al. (2004, p. 331) conclude 'cross-sectional investigations of genetic and environmental influences on aging that can estimate the contributions to the mean level of performance are failing to capture the dynamic process of aging'. In short, by studying crosssectional snapshots as a proxy for development, we may draw incorrect inferences about the most intrinsically developmental component: change itself.

Second, the extent to which genetic and cultural influences differ when modelled using crosssectional versus longitudinal methods may provide a unique source of insight. For instance, the target article discusses the greater risk of skin cancer for European ancestry Australians. However, here too within-person processes are crucial. Among genetically similar individuals, the elevated risk of skin cancer is present almost exclusively in those born in Australia (Olsen et al., 2020). Genetically comparable individuals who move to Australia later in life are at much less elevated risk, suggesting that skin cancer risk is mostly due to early life exposure to the sun, further narrowing down the mechanisms at play. In other words, although cross-sectional data can provide a start of our investigations, it is only by taking a truly developmental perspective that we can hope to truly triangulate mechanisms.

Third and finally, large longitudinal datasets are the most promising source of unique and novel insight. This, combined with the existing dearth of longitudinal sources of information, should encourage funders and scholars alike to better resource larger, collaborative studies that better capture the richness of phenotypic processes, with a special focus on longitudinal, within-person measurements.

Uchiyama et al. make a compelling case that aggregate findings do not generalize to subgroups. The same is true about phenotypic change: Trajectories of heritability are not necessarily informative about heritability of trajectories, yet it is at the level of within-person processes that individual lives unfold. We think the cultural evolutionary behavioural genetics approach would be enriched by making within-person change the core topic of interest. By studying the heritability of trajectories instead of the trajectory of heritability, we may finally, in the words of Molenaar (2004) 'bring the person back, this time forever'. 


\section{Conflict of interest statement}

No conflicts to report.

\section{Funding statement}

This project was supported by funding from the Eunice Kennedy Shriver National Institute of Child Health and Human Development grant P5OHDo52120 (SH) and a Hypatia fellowship (RAK). Views expressed herein are those of the authors and have neither been reviewed nor approved by the granting agencies.

\section{References}

Finkel, D., Davis, D. W., Turkheimer, E., \& Dickens, W. T. (2015). Applying biometric growth curve models to developmental synchronies in cognitive development: The Louisville twin study. Behavior Genetics, 45(6), 600-609. doi: 10.1007/s10519-015-9747-1

Finkel, D., \& Pedersen, N. L. (2004). Processing speed and longitudinal trajectories of change for cognitive abilities: The Swedish Adoption/Twin Study of Aging. Aging Neuropsychology and Cognition, 11(2-3), 325-345. doi: 10.1080/13825580490511152

Hart, S. A., Logan, J. A., Soden-Hensler, B., Kershaw, S., Taylor, J., \& Schatschneider, C. (2013). Exploring how nature and nurture affect the development of reading: an analysis of the Florida Twin Project on reading. Developmental Psychology, 49(10), 1971. doi: 10.1037/ao031348

Hatoum, A. S., Rhee, S. H., Corley, R. P., Hewitt, J. K., \& Friedman, N. P. (2018). Etiology of stability and growth of internalizing and externalizing behavior problems across childhood and adolescence. Behavior Genetics, 48(4), 298-314. doi: 10.1007/s10519-018-9900-8

Logan, J. A., Hart, S. A., Cutting, L., Deater-Deckard, K., Schatschneider, C., \& Petrill, S. (2013). Reading development in young children: Genetic and environmental influences. Child Development, 84(6), 2131-2144. doi: 10.1111/cdev.12104

Lyons, M. J., Panizzon, M. S., Liu, W., McKenzie, R., Bluestone, N. J., Grant, M. D., ... \& Xian, H. (2017). A longitudinal twin study of general cognitive ability over four decades. Developmental Psychology, 53(6), 1170. doi: 10.1037/devo000303

Molenaar, P. C. (2004). A manifesto on psychology as idiographic science: Bringing the person back into scientific psychology, this time forever. Measurement, 2(4), 201-218. doi:

10.1207/s15366359mea0204_1

Olsen, C. M., Pandeya, N., Law, M. H., MacGregor, S., lles, M. M., Thompson, B. S., ... \& OSkin Study. (2020). Does polygenic risk influence associations between sun exposure and melanoma? A prospective cohort analysis. British Journal of Dermatology, 183(2), 303-310. doi: 10.1111/bjd.18703

Reynolds, C. A., Finkel, D., Gatz, M., \& Pedersen, N. L. (2002). Sources of influence on rate of cognitive change over time in Swedish twins: An application of latent growth models. Experimental Aging Research, 28(4), 407-433. doi: 10.1080/03610730290103104

Zhang, C., \& Pierce, B. L. (2014). Genetic susceptibility to accelerated cognitive decline in the US Health and Retirement Study. Neurobiology of Aging, 35(6), 1512-e11. doi:

10.1016/j.neurobiolaging.2013.12.021 